\title{
Wer webt den Rechtsstoff? - Kodifikationsdebatten als Debatten um Macht und Deutungshoheit im Recht
}

\section{Arbeitsgruppe auf der Sommerakademie der Studienstiftung des deutschen Volkes und des Max-Weber-Programms in Ftan 2012}

Stefan Lenz

Seit über 20 Jahren ist es erklärtes politisches Ziel, dass die Harmonisierung des Privatrechts durch den europäischen Gesetzgeber eines Tages in ein Gemeinsames Europäisches Zivilgesetzbuch mündet. Die Kodifizierung des Privatrechts zählt freilich zu den umstrittensten Fragen der europäischen Rechtspolitik. Neu ist eine solche Kodifikationsdebatte nicht: Erstmals im 17. Jahrhundert kam der Gedanke auf, ein Rechtsgebiet gehöre systematisch und vollständig in einem einzigen Gesetzbuch geregelt. Kaum waren um 1800 die ersten Kodifikationen ins Werk gesetzt, regte sich massiver Widerstand. Am wirkungsvollsten formulierte ihn Savigny, als er sich im Kodifikationsstreit von 1814 gegen Thibauts Vorschlag eines Bürgerlichen Gesetzbuches für den Deutschen Bund wandte. Was lässt sich aus den Debatten der Vergangenheit für die Kontroverse der Gegenwart lernen, ob ein Gemeinsames Europäisches Zivilgesetzbuch wünschenswert ist? Diesem Zusammenhang ging dieses Jahr eine Arbeitsgruppe auf der Sommerakademie der Studienstiftung des deutschen Volkes und des Max-Weber-Programms im schweizerischen Ftan nach. Zwei Wochen lang widmeten sich zwölf Studentinnen und Studenten der Rechtswissenschaft gemeinsam mit ihren Dozenten Christiane Wendehorst und Thomas Simon (beide Wien) in Referaten, Diskussionen und Gruppenarbeiten den rechtlichen, historischen und politischen Facetten des Kodifikationsgedankens.

Den Auftakt machte Donatella Angino (Augsburg), indem sie ein Gemälde der traditionellen Rechtsquellenlandschaft der Frühen Neuzeit entwarf. Die auf den ersten Blick unzähligen Quellen der vormodernen Rechtsordnung ließen sich auf drei Ursprünge zurückführen: Gewohnheit, Wissenschaft und Gesetzgebung. Bis ungefähr 1800 sei das Recht größtenteils nicht in staatlicher Vorkehrung gewesen. Der Erlass der drei sog. naturrechtlichen Kodifikationen zu dieser Zeit (ALR 1794, Code Civil 1804 und ABGB 1811) bedeute deshalb eine Zäsur für die Rechtsgeschichte. Angekündigt habe sich der Einschnitt durch den schleichenden Wandel von usualem zu formellem Rechtsgeltungsverständnis: Die Zeitgenossen akzeptierten nicht mehr ausschließlich die Observanz, d.h. die langandauernde Übung als Geltungsgrund des Rechts, sondern auch den Formalakt des Inkraftsetzens, d.h. den bloßen Willen des staatlichen Gesetzgebers. Dieser Umschwung erst habe das staatliche Gesetz durchsetzungsfähig und eine Kodifikation überhaupt vorstellbar gemacht.

* Stefan Lenz ist studentische Hilfskraft am Kommunalwissenschaftlichen Institut der Westfälischen Wilhelms-Universität Münster. 
Den Begriff der Kodifikation, 1815 von Bentham eingeführt, klärte das Referat von Kilian Wolf (München). Er identifizierte die Einheitlichkeit des Rechts als Kernanliegen von Kodifikationen. Auf drei Ebenen werde sie angestrebt: auf systematischtechnischer Ebene, weil das Recht vollständig und nach einheitlichem Muster geregelt sei; auf sozialer Ebene, weil jeder vor dem Recht gleich sei; auf territorialer Ebene, weil das Recht für ein ganzes Gebiet gelte. Kodifikationen unterschieden sich von anderen Versuchen, das Recht zu systematisieren, etwa in Lehrbüchern und sog. Privatkodifikationen: Formal verdankten Kodifikationen ihre Geltung staatlichem Befehl; materiell leisteten sie einen Beitrag zur inneren Staatswerdung und bildeten den Ausgangspunkt nationaler Rechtsgeschichte. Nach einem Blick auf ALR, Code Civil und ABGB kam der Referent zum Schluss, dass eine Kodifikation immer auch Rechtserneuerung bedeute, die Vorstellung also ein Trugschluss sei, man könne darin bloß sammeln, was ohnehin schon gelte und praktiziert werde. Dass eine Kodifikation eine Revolution bedeute, wollte er damit noch nicht gesagt haben; vielmehr bestünden Elemente von Kontinuität und Diskontinuität nebeneinander.

Die Richter sahen sich durch Kodifikationen in ihrem Rollenverständnis herausgefordert, wie Florian Gröntgen (Bonn) aufzeigte. Zuvor hätten die Juristen Recht selbst geschöpft; unter einer Kodifikation fürchteten sie zu mechanischen Rechtsanwendern degradiert zu werden. Rechtsfindungsregeln seien Versuche gewesen, die Bindung des Richters an das Gesetz gegen Widerstände durchzusetzen: Die Auslegung von Kodifikationen sei bei Androhung von Strafe verboten gewesen; sei der Richter auf eine Lücke gestoßen, so habe er den Fall bei Hofe vorlegen müssen („,référé législatif“). Auf diese Vorschriften habe der Gesetzgeber verzichtet, als er das praktische Bedürfnis nach gewissem Spielraum der Justiz erkannt hätte. Die Diskussion konzentrierte sich auf moderne Äquivalente zu Rechtsfindungsregeln: Regelbeispielstechnik, bewegliches System und die Kombination von rigiden Sondertatbeständen mit einem Auffangtatbestand. Deutlich wurde, dass alle Versuche zum Scheitern verurteilt sind, den Richter zum „Mund des Gesetzes“ (Montesquieu) zu machen: Rechtsanwendung beinhaltet stets Rechtsauslegung. Wohl aber bereiten solche Regelungstechniken dem Richter gesteigerte Begründungslasten bei der Auslegung - einen methodenbewussten Juristenstand vorausgesetzt.

Furcht vor Bedeutungsverlust machte Stefan Lenz (Münster) auch als ein Motiv Savignys im prototypischen Kodifikationsstreit mit Thibaut aus. Savigny habe die Autonomie des Rechts gegenüber der Politik wahren wollen; innerhalb des Rechts habe er den Primat der Wissenschaft über die Rechtsprechung verteidigt. Sein zentraler Einwand sei gewesen, dass in Zeiten von Aufstieg oder Niedergang eines Volkes die sprachlichen und wissenschaftlichen Voraussetzungen zur Kodifikation fehlten und in Zeiten der Blüte das Bedürfnis. Im schlimmsten Fall schade eine Kodifikation, im besten Fall sei sie überflüssig. Über die Ziele von Rechtssicherheit und Rechtseinheit 
sei sich Savigny mit Thibaut einig gewesen; auseinandergegangen seien ihre Meinungen über den besten Weg dorthin. Als Gegenentwurf habe Savigny die historische Rechtswissenschaft präsentiert, die das Recht in Deutschland zu neuer Blüte führen könne. Als bleibende Leistung der Historischen Schule wurde in der Diskussion ausgemacht, die deutschen Juristen nachdrücklich auf die methodengeleitete Exegese von Texten verpflichtet zu haben: zunächst der Digesten, später des BGB.

Annabelle Meier (Würzburg) richtete den Blick nach Österreich, wo ungeachtet des Aufstiegs der Historischen Schule zunächst das naturrechtiche ABGB im Fokus der Wissenschaft gestanden habe, bis Mitte des 19. Jahrhunderts Unterrichtsminister Leo von Thun-Hohenstein die österreichische Jurisprudenz auf den Kurs der Pandektenwissenschaft gezwungen habe. Instrumente dieser „Revolution von oben“ sei neben einer Studienreform und veränderter Berufungspolitik die Neuinterpretation des ABGB im pandektistischen Sinne gewesen. Abgesehen von offiziell geäußerten wissenschaftlichen Gründen hätten insgeheime politische Motive eine Rolle gespielt: Im Inneren habe man den Studenten eine konservative Haltung angewöhnen, nach außen die Stellung Österreichs im Deutschen Bund durch ein Signal der Modernisierung stärken wollen. Wie sich in der Diskussion herauskristallisierte, reichen die Nachwirkungen der „Pandektenkur“ so weit, dass durch die Wissenschaft transportiertes Gewohnheitsrecht Teile des ABGB beherrscht. Damit ist in Österreich möglicherweise die kryptische Vorhersage Savignys eingetreten, wonach eine Kodifikation leicht von einer „eigentlich regierenden Rechtsquelle“ überschattet werde.

Die Frage nach einer weiteren Rechtsquelle neben der Kodifikation vertiefte Wienke Werner (München), indem sie dem sog. Richterrecht nachging. Einerseits verbinde sich mit Kodifikationen der Anspruch, dass sie vollständig seien und der Richter das Gesetz ausschließlich anwende; das gelte erst recht in der gewaltenteiligen Demokratie. Andererseits bringe Normanwendung im Einzelfall stets neue Fallnormen hervor und könne keine Kodifikation auf Dauer lückenlos sein; das zwinge zur richterlichen Rechtsfortbildung. Die Referentin grenzte Richterrecht vom Gewohnheitsrecht anhand der Begründungsmuster ab: Ersteres suche den größtmöglichen argumentativen Anschluss an die Kodifikation, letzteres beanspruche Geltung aus sich selbst heraus. Die Diskussion kreiste um die Frage, ob Richterrecht tatsächlich eine eigene Rechtsquelle ist. Das ist aus einer externen Perspektive zu bejahen, welche die rechtlichen Entscheidungen in einem System von außen beschreibt; aus einer internen Perspektive dagegen, der es um die richtige Dogmatik des positiven Rechts geht, erlangt Richterrecht Rechtscharakter allenfalls vermittelt über die Verfassungsgrundsätze von Vertrauensschutz und Gleichbehandlung.

Die Brücke vom historischen zum gegenwartsorientierten Teil der Sommerakademie baute Konstantin Sauer (Augsburg). Er stellte dem kontinentaleuropäischen Civil Law das englische Common Law gegenüber, in dem es nie zu einer Kodifikation 
gekommen sei und dem seine Verträglichkeit mit einem Gemeinsamen Europäischen Zivilgesetzbuch deshalb vielfach abgesprochen werde. In England sei dem Richter ausdrücklich die Rolle eines Rechtsschöpfers zugewiesen, während der parlamentarische Gesetzgeber lediglich punktuell tätig werde, um ein politisches Regelungsziel zu erreichen - oder in neuerer Zeit, um europäische Richtlinien umzusetzen. Der Referent ließ Zweifel aufkommen an der gängigen Annahme, dass beide Rechtskreise kategorial verschieden seien: Genauso wie Kodifikationen auf dem Kontinent sei auch die Präjudizientreue der Common-Law-Richter vergleichsweise neu. Kalifornien hat sogar ein Common-Law-System, dessen Zivilrecht kodifiziert ist, ohne dass es dadurch zu einer Civil-Law-Rechtsordnung geworden wäre. Denn wie in der Diskussion betont wurde, gilt statt eines Gesetzes das dazugehörige Fallrecht als Rechtsquelle, sobald erste Urteile ergangen sind.

Die folgenden beiden Referate versicherten die Arbeitsgruppe der Grundlagen des Unionsrechts. Eduardo Mori Monteiro (Heidelberg) präsentierte in Entsprechung zum Auftaktreferat die Rechtsquellenlandschaft der Europäischen Union: das Primärrecht aus Verträgen, Grundrechtecharta und allgemeinen Rechtsgrundsätzen; das Sekundärrecht hauptsächlich aus Verordnungen und Richtlinien. Die Rolle des EuGH beschrieb er als die eines „Hüters“ (Carl Schmitt) nicht bloß der Verträge, sondern aller Quellen des Unionsrechts. Johannes Zehendner (Augsburg) ging darauf ein, wie die Mitgliedsstaaten Richtlinien in ihren kodifikationsgeprägten Rechtsordnungen umsetzen können, sei es etwa durch Parallel- oder Sondergesetzgebung oder die Integration in die Kodifikation. In der Diskussion wurde betont, dass vollharmonisierende Richtlinien der Verordnung zwar angenähert sind, aber nach wie vor nennenswerte Unterschiede bestehen. Denn erstere stellten den Mitgliedsstaaten die Modalität der Umsetzung und Gesetzgebung außerhalb des sachlich harmonisierten Bereichs anheim. Erwogen wurde auch, ob die Bindungswirkung von EuGH-Präjudizien erga omnes als eine faktische richtig beschrieben ist oder ob es sich nicht doch um eine - wenn auch vermittelte - rechtliche Bindung handelt, weil Rechtsauslegung wider den EuGH mit dessen Auslegungsmonopol und der mitgliedsstaatlichen Loyalitätspflicht kollidiert.

Kristin Bubr (Heidelberg) beleuchtete die in den 80er Jahren einsetzenden Bemühungen von Wissenschaftlergruppen, das Europäische Privatrecht in Regelform zu systematisieren. Diese Versuche seien als Vorarbeiten für ein Gemeinsames Europäisches Zivilgesetzbuch zu verstehen. Die sog. Privatkodifikationen seien ihrerseits nicht ohne historische und internationale Vorläufer: Corpus Iuris Civilis, Sachsenspiegel, Restatements des American Law Institute. Die Referentin konzentrierte sich auf den DCFR, dem Kritiker vorhielten, er sei unter fragwürdigem politischem Einfluss entstanden und erlange eine faktische Autorität, die ihm mangels eines Gesetzgebungsbeschlusses nicht zustehe. In lebhafter Diskussion wurde angemahnt, dass 
Ansatzpunkt der rechtswissenschaftlichen Kritik nicht die Akzeptanz von Privatkodifikationen sein darf, sondern bloß deren Qualität - die in der Tat zu wünschen übrig lässt. Zweifel wurden laut, ob eine politikferne Konzeption von Rechtswissenschaft, wie sie schon Savigny vorschwebte, im demokratischen Verfassungsstaat überhaupt praktikabel und erstrebenswert ist. Die Wissenschaft hat vielmehr politische Regelungsziele zu akzeptieren und technisch umzusetzen; im Gegenzug muss die Politik ausreichend Zeit lassen und Einwände aus der Wissenschaft ernst nehmen.

Isabella Beck (München) machte eine begriffliche Unterscheidung explizit, die schon länger im Raum gestanden hatte, nämlich zwischen kodifizierender und steuernder Gesetzgebung. Idealtypisch halte eine Kodifikation bloß das Recht nachträglich fest, das in Rechtsprechung und Wissenschaft ohnehin schon praktiziert werde, während Steuerungsgesetzgebung dazu diene, ein einzelnes politisches Ziel notfalls kontrafaktisch durchzusetzen. Die Referentin lenkte das Augenmerk auf den Paradigmenwechsel von Mindest- zu Vollharmonisierung und daher rührende methodische Probleme. Mehr als bisher drohe die gespaltene Auslegung von Kodifikationen: Zum Beispiel dürfe eine nationale Vorschrift vielfach weder teleologisch reduziert noch analog angewandt werden, wenn sie auf einer vollharmonisierenden Richtlinie mit einem typisierenden Schutzkonzept beruhe. Kriterium für die methodische Ungleichbehandlung sei allein der - freilich nicht offensichtliche - Normhintergrund. Einbußen an Kohärenz, Übersichtlichkeit und Rechtssicherheit seien programmiert.

Matthias Reidt (Köln) band in seinem Referat die Fäden zusammen und beantwortete die Gretchenfrage, wie er es mit dem Gemeinsamen Europäischen Zivilgesetzbuch halte. Eingangs zeigte er erstaunliche Parallelen zwischen dem Kodifikationsstreit von 1814 und der gegenwärtigen Kontroverse auf: Viele Argumente entsprächen sich nicht bloß inhaltlich, einige kämen sogar in ganz ähnlichem rhetorischem Gewande daher. Heute werde das Vorhaben einer Zivilkodifikation im Kern damit legitimiert, dass sie für die Vollendung des Binnenmarktes unabdingbar sei. Der Referent zweifelte, ob nicht weniger unterschiedliche Zivilrechte als andere Hindernisse den Binnenmarkt lähmen, seien es etwa Sprachbarrieren oder generelle Skepsis von Verbrauchern gegenüber ausländischen Produkten. Das ungeklärte Bedürfnis eines gemeinsamen Marktes nach einem gemeinsamen Recht sei nicht die einzige offene Frage: Eine Zivilkodifikation träfe auf divergierende nationale Rechtskulturen, eine unvorbereitete Rechtspraxis und habe bislang nicht einmal eine Kompetenzgrundlage in den Verträgen. Die mangelhafte Qualität europäischer Rechtssetzung schließlich, die Zeitdruck und politischem Einfluss geschuldet sei, lasse für das Niveau einer Kodifikation nichts Gutes erwarten. Für ein Gemeinsames Europäisches Zivilgesetzbuch sei die Zeit nicht reif - ein Fazit, das in der Arbeitsgruppe breite Zustimmung erntete. 
Eben jene Qualitätsfragen, auf denen ein Gutteil der Skepsis beruht, waren Gegenstand zweier Gruppenarbeitsphasen. Ausgehend von der Verbraucherrechte-Richtlinie leuchteten die Studentinnen und Studenten zuerst Alternativen zum gegenwärtigen Verbraucherbegriff und zum Informationsmodell aus genauso wie Möglichkeiten, Informationspflichten und Widerrufsrechte in Kodifikationen zu integrieren. Als Basis dienten Hans-W. Micklitz' Thesen für den 69. Deutschen Juristentag 2012 und die Vorschläge des Autorenkollektivs Eidenmüller/Faust/Grigoleit/Jansen/Wagner/Zimmermann zur „Revision des Verbraucher-acquis“. In der zweiten Phase, moderiert von Isabella Beck, stand der Entwurf eines Gemeinsamen Europäischen Kaufrechts auf dem Prüfstand. Neben seiner Regelungstechnik war Thema, ob sein Erlass mit dem Subsidiaritäts- und Verhältnismäßigkeitsprinzip vereinbar wäre - was trotz Zweifeln an der Effektivität des Instruments bejaht wurde.

Am Ende der Sommerakademie stand die Einsicht, dass Kodifikationen als Markenzeichen des kontinentaleuropäischen Rechts keineswegs selbstverständlich sind und damit weder die Rollenaufteilung zwischen parlamentarischem Gesetzgeber und Juristenstand, noch die Idee der systematischen Geschlossenheit und Vollständigkeit des Rechts oder auch bloß des staatlichen Anwendungsbefehls als hinreichendem Geltungsgrund. Das Bewusstsein hierfür gilt es in Forschung und Lehre zu schärfen. Besonders für alle, die mit dem Europäischen Privatrecht befasst sind, lohnt der Blick zurück auf die scheinbar fernen Debatten des 19. Jahrhunderts; umgekehrt werden Rechtshistoriker sich im Kodifikationsstreit von heute der Zeitlosigkeit ihrer Fragen gewahr. Der Erkenntnisgewinn in der Arbeitsgruppe jedenfalls bestätigte das Konzept von Studienstiftung und Max-Weber-Programm, auf ihren Sommerakademien Raum zu schaffen für wissenschaftliche Diskussionen, wie sie in dieser Intensität im Studienalltag kaum möglich sind. 